\title{
USO DE LA INTELIGENCIA COMPETITIVA EN LOS PROCESOS DE COLABORACION EN EL SECTOR FARMACEUTICO ESPAÑOL
}

\author{
Using competitive intelligence in collaborative \\ processes in the Spanish pharmaceutical sector
}

María-Paula Fernández-Arias, Pilar Quevedo-Cano y Antonio Hidalgo-Nuchera

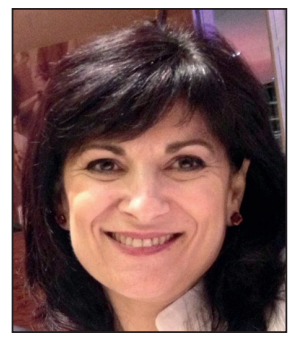

María-Paula Fernández-Arias es profesora de organización de empresas en la Escuela Técnica Superior de Ingeniería de Sistemas Informáticos de la Universidad Politécnica de Madrid (UPM), licenciada en ciencias económicas y empresariales y actuaria de la empresa financiera y de seguros por la Universidad Complutense de Madrid. Responsable del módulo de gestión de empresas del Máster de Acupuntura y Moxibustión en el Colegio Oficial de Médicos de Madrid. Realiza su tesis doctoral sobre la gestión de la inteligencia competitiva en el sector farmacéutico español. http://orcid.org/0000-0001-6239-1834

Universidad Politécnica de Madrid, ETSI de Sistemas Informáticos Dpto. Ingeniería de Organización, Administración de Empresas y Estadística Alan Turing, s/n, Campus Sur, 28031 Madrid, España pfarias@etsisi.upm.es

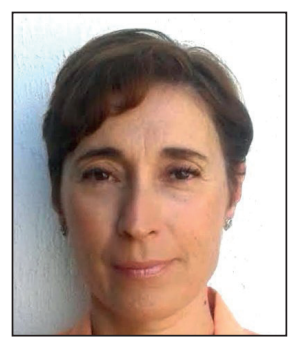

Pilar Quevedo-Cano, profesora de organización de empresas en la Escuela Técnica Superior de Ingeniería de Sistemas Informáticos de la Universidad Politécnica de Madrid (UPM), es doctora en ciencias económicas y empresariales por la Universidad Complutense de Madrid. Ha participado en proyectos financiados por la Unión Europea en el área de innovación tecnológica. Sus líneas de investigación están relacionadas con la gestión de la edad en la empresa.

http://orcid.org/0000-0003-4710-8484

Universidad Politécnica de Madrid, ETSI de Sistemas Informáticos Dpto. Ingeniería de Organización, Administración de Empresas y Estadística Alan Turing, s/n, Campus Sur, 28031 Madrid, España pquevedo@etsisi.upm.es

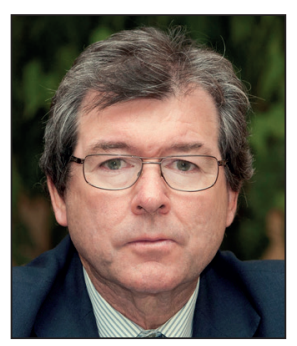

Antonio Hidalgo-Nuchera es catedrático de organización de empresas en la Escuela Técnica Superior de Ingenieros Industriales de la Universidad Politécnica de Madrid (UPM) y director del Grupo de Investigación en Innovación, Propiedad Industrial y Política Tecnológica (Innopro). Ha participado en proyectos financiados por la Comisión Europea y organismos nacionales sobre innovación tecnológica, transferencia de tecnología y desarrollo de políticas tecnológicas.

http://orcid.org/0000-0002-3598-9862

Universidad Politécnica de Madrid, ETSI Industriales Dpto. Ingeniería de Organización, Administración de Empresas y Estadística José Gutiérrez Abascal, 2. 28006 Madrid, España antonio.hidalgo@upm.es

\section{Resumen}

En el sector farmacéutico se ha generado en los últimos años todo tipo de acuerdos con el propósito de explorar nuevas vías de crecimiento. La inteligencia competitiva (IC) se revela crucial en estos procesos para mejorar las relaciones de colaboración. Se presenta el resultado de un estudio realizado a una muestra de 186 compañías farmacéuticas españolas para analizar cómo utilizan la IC con fines cooperativos. Las compañías farmacéuticas utilizan el ciclo de IC tanto para extraer inteligencia ofensiva y defensiva como, cada vez más, para obtener inteligencia cooperativa. Buena parte de las empresas usan la IC para buscar socios con el fin de compartir fuentes de información, formas de financiación o proyectos de innovación. Este resultado indica la importancia de potenciar y adaptar las técnicas de IC a las nuevas demandas de información dado que cada vez se utilizan en mayor medida en los procesos con fines cooperativos que están reestructurando el sector.

\section{Palabras clave}

Inteligencia competitiva; Ciclo de IC; Cooperación; Redes de información; Redes de cooperación; Difusión de conocimiento. 


\begin{abstract}
It is quite common to find competitive intelligence $(\mathrm{Cl})$ activities in pharmaceutical companies. These companies use the $\mathrm{Cl}$ process, not only to act against competitors, a traditional goal of the $\mathrm{Cl}$, but also with the purpose of enhancing cooperation. This paper proposes that this trend should be deeply analyzed so that IC techniques can be adapted to the new demands of information. $\mathrm{Cl}$ cycle is used to extract offensive intelligence, defensive intelligence, and, more increasingly, cooperative intelligence. The results of a study developed with a sample of 186 Spanish pharmaceutical companies are presented. The main goal was to analyze the extent to which these companies use $\mathrm{Cl}$ processes for cooperative purposes. Results confirm that companies use $\mathrm{Cl}$ tools for finding partners to share information sources, funding sources, or to carry out innovation projects.
\end{abstract}

\title{
Keywords
}

Competitive intelligence; Cycle IC; Cooperation; Coopetition; Information networks; Dissemination of knowledge.

Fernández-Arias, María-Paula; Quevedo-Cano, Pilar; Hidalgo-Nuchera, Antonio (2016). "Uso de la inteligencia competitiva en los procesos de colaboración en el sector farmacéutico español”. El profesional de la información, v. 25, n. 5, pp. 778-786.

https://doi.org/10.3145/epi.2016.sep.08

\section{Introducción}

En los últimos años estamos asistiendo a un aumento de los procesos de integración entre las empresas del sector farmacéutico (fusiones, adquisiciones y acuerdos de colaboración). En este sentido, según los informes de Bloomberg, 2014 fue un año record. En 2014 y 2015 asistimos al establecimiento de todo tipo de acuerdos entre empresas tan importantes como Pfizer, Merk, Shire, Actavis, Allergan, etc., en un intento de explorar nuevas vías de crecimiento.

Una de las técnicas en las que estas empresas han sido pioneras, la inteligencia competitiva (IC), se revela crucial en esos procesos para mejorar las relaciones de colaboración. Tales empresas no sólo utilizan la IC para actuar frente a los competidores, objetivo tradicional de la misma, sino que cada vez más la utilizan en la búsqueda de información y conocimiento para potenciar la cooperación.

La asociación Strategic and Competitive Intelligence Professionals (SCIP) define la IC como el proceso de recopilar éticamente, analizar y diseminar inteligencia segura, relevante, específica, a tiempo, previsora y utilizable en el entorno de los negocios, los competidores y la propia organización (SCIP, 1999).

En la bibliografía existen otras muchas definiciones de inteligencia competitiva (de inteligencia estratégica y de inteligencia económica) que intentan diferenciar los conceptos que han ido surgiendo en torno a esta disciplina y que, en muchas ocasiones, son empleados como sinónimos (Cohen, 1997; McGonagle; Vella, 1998; Bernhardt, 1994; etc.). Los sectores pioneros en IC fueron los de comunicaciones, farmacéutico, aeroespacial y defensa (Tena; Comai, 2005; Hidalgo-Nuchera; Iglesias-Pradas; Hernández-García, 2009).

La IC aporta información en la que basar la planificación estratégica y la toma de decisiones y a la vez tiene un impacto en el rendimiento de la organización (Prescott, 1999; Herring, 1999, Ortoll et al., 2010; Antia; Hesford, 2007; Tena; Comai, 2003).
La importancia dada a la IC se ha basado históricamente en las actividades ofensivas y defensivas que potencia en la empresa. Sin embargo, algunos autores (Nalebuff; Brandenburger, 1997; Salvetat; Le-Roy, 2007; Cavaller, 2009) han abierto otra línea de investigación afirmando que cada vez es más habitual encontrar empresas que ponen en marcha su proceso de IC con el objetivo de potenciar mejoras internas o colaboraciones con otras empresas (por ejemplo, para líneas de open innovation y de coopetition).

Así, Cavaller (2009) insta a implementar y sostener procesos de gestión de información y de conocimiento, tales como la IC, con el fin de promover la interacción entre los agentes con los que tiene que negociar para alcanzar sus objetivos estratégicos. El uso de la IC más allá de sus fines tradicionales, desarrolla otras formas de hacer inteligencia y descubre a los profesionales de la información otros objetivos compatibles con los que hasta ahora manejaban.

\section{Muchas empresas usan la IC para buscar socios con el fin de compartir fuentes de información, formas de financiación o proyectos de innovación}

Los procesos de integración que ha vivido el sector farmacéutico, antes citados, han hecho necesario el manejo de una gran cantidad de información por parte de los profesionales. Por este motivo éstos usan procesos que originalmente sirvieron para conseguir inteligencia competitiva $y$ que ahora usan para buscar inteligencia colaborativa.

El objetivo de este trabajo, en línea con lo anteriormente expuesto, es analizar hasta qué punto las empresas del sector farmacéutico español utilizan la inteligencia competitiva con fines cooperativos. Para ello se hace un estudio empírico en una muestra representativa de este sector, en concreto, en el conjunto de empresas que integran Farmaindustria, la asociación que agrupa a las principales empresas del mismo. 
Para conseguir nuestro objetivo estructuraremos el trabajo como sigue: en primer lugar, expondremos el modelo de las cinco fases en el ciclo de IC, a través del cual explicaremos el proceso de generación de la inteligencia en las compañías. Siguiendo las sugerencias de Cetisme, 2003; Jakobiak, 1992; Martinet; Martí, 1995, Escorsa; Maspons, 2001; Ashton; Stacey, 1995; Hidalgo-Nuchera et al., 2002, se incorporan las acciones que se derivan del uso de la inteligencia competitiva como parte fundamental de dicho proceso. En segundo lugar, analizaremos la utilización que hacen de la IC las empresas de la muestra con fines cooperativos. Por último se expondrán las conclusiones del trabajo y las líneas que éste abre, y se explorarán posibles futuros trabajos.

\section{Fases del ciclo de la IC}

Tradicionalmente, la generación de inteligencia se entiende como un proceso iterativo estructurado en las cuatro fases siguientes (Ortoll et al., 2010; Prescott, 1999; Choo, 2002; Lesca; Caron-Fasan, 2006; Arroyo-Varela, 2005; Vignettes, 2010):

1) organización e identificación de las necesidades de información;

2) búsqueda y recogida de información;

3) análisis e interpretación de la información;

4) difusión basada en la información analizada.

Otros autores (Cetisme, 2003; Jakobiak, 1992; Martinet; Martí, 1995; Escorsa; Maspons, 2001; Ashton; Stacey, 1995, Hidalgo-Nuchera et al., 2002) siguen el mismo esquema descrito pero con algunas diferencias y determinan añadir una fase más, la acción. Para ellos es imprescindible que la toma de decisiones termine en acciones concretas en la organización susceptibles de ser controladas y mejoradas. Por otra parte, aunque las actividades aparecen como una secuencia, en realidad, están conectadas a través de una retroalimentación continua (Tena; Comai, 2003).

El modelo de ciclo de IC que usaremos para el análisis considera las fases tradicionales, incorpora la fase descrita por los últimos autores como "acción" y añade explícitamente las

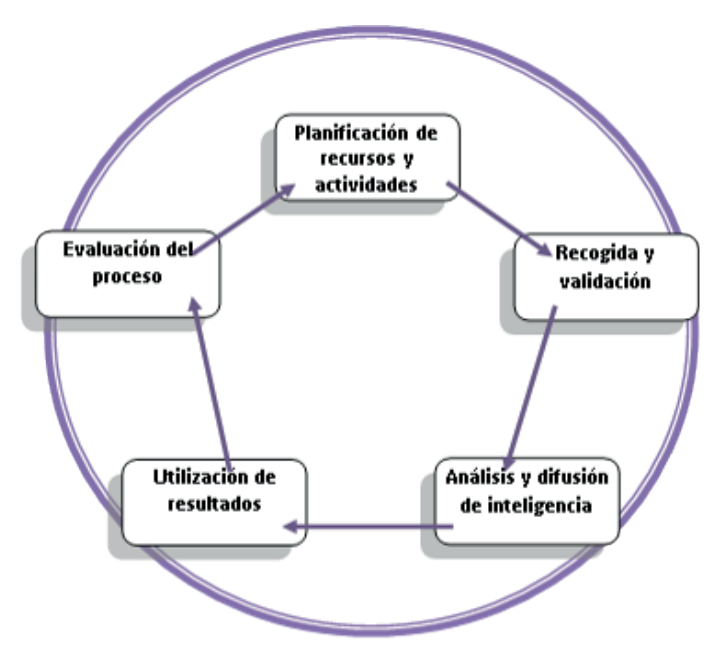

Figura 1. Ciclo tradicional de la IC actividades de "evaluación" del proceso en su última fase. Esta última nos parece la base de la mejora continua del proceso (figura 1).

\subsection{IC y cooperación}

Podemos clasificar la IC según los objetivos para los que se diseñe (Tena; Comai, 2001; Hussey; Jenster, 1999). La empresa puede determinar que una parte o toda su actividad de IC persiga realizar la llamada "inteligencia competidora" (para realizar acciones defensivas y ofensivas en el mercado), y puede determinar que una parte o toda su IC se destine a una "inteligencia cooperativa", entendiendo ésta como la IC que trabaja en dos direcciones: una explícita que busca acuerdos, alianzas, etc., y otra tácita que sigue la conducta de las empresas del sector con el objetivo de mantener la estabilidad en éste. Este tipo de inteligencia busca obtener un beneficio para todas las compañías implicadas (Tena; Comai, 2001).

Diversos autores han explorado la importancia de las redes de cooperación en los procesos de IC (Ortoll et al., 2010; Palop; Vicente, 1999; Escorsa; Maspons, 2001; Trim, 2004) concluyendo que el impacto de la cooperación en la eficiencia de todo el proceso de IC es significativo.

\section{Es frecuente la colaboración entre clien- te y proveedor pero ha sido más difícil hasta hace algunos años hallar casos de colaboración con los competidores}

Las redes de cooperación son fuente de creación de valor en muchos ámbitos de la organización y es imprescindible que el profesional optimice sus actividades hacia estas redes (Nalebuff; Brandenburger, 1997; Salvetat; Le-Roy, 2007; Prescott, 2003). En este sentido, en estudios posteriores, podría profundizarse en el concepto de coopetition y en su potencial para la creación de valor. El concepto excede los objetivos de este trabajo pero comparte la idea básica de las relaciones de colaboración por parte de agentes que tradicionalmente no son colaboradores.

Ortoll et al. (2010) definen tres fases útiles para el profesional de la IC cuando debe enfocar su actividad con el objetivo de facilitar la cooperación y la búsqueda de creación de valor: organización de la IC, búsqueda de información, análisis e interpretación.

Así, en la primera fase, la organización debe elaborar un mapa de los vínculos de su red de cooperación (personas, características de los posibles socios, posibles enlaces en su organización, etc.). En el diseño de su plan de cooperación debe estar previsto el nivel de compromiso con los socios, el nivel de confidencialidad de la información, los sistemas de protección de la información, y la garantía de seguridad de la información interna.

En la segunda fase, proceso de búsqueda, es necesario considerar la red de cooperación como una fuente más de información, con sus características y enlaces.

En la tercera fase, análisis e interpretación de los datos, se deben establecer protocolos de análisis e interpretación 
para la información recibida de los socios cooperantes, al igual que son necesarios cuando se obtiene por otras fuentes.

En general, cuando se habla de cooperación en el marco de la IC, en la bibliografía se encuentran algunas referencias a la colaboración con el cliente y con el proveedor (Palop; Vicente, 1999) pero ha sido más difícil hasta hace algunos años hallar propuestas de colaboración desde la IC hacia los competidores. A pesar de que algunos autores hablan de esa posibilidad dentro de la IC (Tena; Comai, 2001; García-Alsina; Ortoll, 2012) aún faltan estudios empíricos que lo respalden.

Del análisis de la bibliografía existente sobre el tema podemos concluir que el uso de la IC para potenciar los beneficios de la cooperación es interesante para las empresas.

No obstante falta evidencia empírica acerca de si se está utilizando esta "inteligencia cooperativa" en la práctica empresarial. Es en esta carencia donde se encuadra nuestro trabajo ya que las compañías farmacéuticas afirman que sí tienen interés en el uso de la IC para cooperar, y en algunas fases del proceso de generación de inteligencia ya lo están haciendo; es necesario ahondar en estas afirmaciones.

Gráficamente (figura 2) encontraríamos el ciclo de la IC actuando en cada fase condicionado por la finalidad perseguida por la empresa (Tena; Comai, 2001).

\section{El sector farmacéutico}

En este trabajo nos centraremos en uno de los sectores pioneros en el desarrollo y uso de la IC, el farmacéutico. Entre los sectores considerados de alta tecnología es uno de los más representativos en cuanto a la necesidad de información y conocimiento debido a su constante innovación en procesos y productos, lo que le lleva a invertir en IC para una mayor eficiencia en sus investigaciones (Baiget, 1999; Tena; Comai, 2001; Escorsa; Maspons, 2001).

En el informe de la Organización Médica Colegial (OMC) de 2014 sobre el sector farmacéutico se dibuja el marco de actuación de esta industria. Así, en los últimos 40 años ha sido el sector con más rápido crecimiento en Europa y Estados Unidos.

La industria de la salud está presente en todas las formas y procesos de globalización, y sus mercados de bienes, manufacturas y servicios, así como sus factores de producción se han mundializado en las últimas décadas de manera vertiginosa. La inversión en I+D en productos farmacéuticos y biotecnología no se ha resentido de forma significativa en Estados Unidos, Europa y Japón a pesar de la crisis económica de 2008 (EU Industrial R\&D Investment Scoreboard, 2010).

Por otra parte, diversos organismos (OMC, 2014) recomien- dan una mayor cooperación entre las empresas del sector y con las administraciones públicas para mejorar su productividad, su control y los beneficios para toda la sociedad y proponen medidas para aumentar la competitividad del sector de alta tecnología farmacéutica.

En esta línea y desde un punto de vista de posible reordenación estratégica de este sector es razonable esperar, con una visión global y competitiva, que continúe el proceso iniciado de concentración a través de alianzas, joint ventures o fusiones en la creación de compañías farmacéuticas solventes e innovadoras, con capacidad de crecimiento orgánico e internacionalización acorde con un sector estratégico, dentro de la economía productiva. Ésta es la tendencia real que están mostrando las compañías del sector en los últimos dos años y que se refleja en las noticias de prensa económica diaria.

\section{El sector farmacéutico destaca en la ne- cesidad de información y conocimiento debido a su constante innovación en procesos y productos}

También es necesario destacar que el modelo de investigación de la industria farmacéutica sigue evolucionando en la última década, desde el patrón clásico de I+D al prototipo denominado I+C (investigación + cooperación y/o colaboración), que se materializa en el aumento de contrataciones externas en universidades, centros hospitalarios, etc.

\section{Metodología}

Para el estudio se decidió tomar como muestra representativa del sector, las empresas adheridas a la Asociación $\mathrm{Na}$ cional Empresarial de la Industria Farmacéutica (Farmaindustria), de España, ya que agrupa a la gran mayoría de laboratorios farmacéuticos. 
Los 186 laboratorios asociados a Farmaindustria, representan en términos de ventas el $84 \%$ del mercado de prescripción y por su número, el $42 \%$ de los titulares de autorizaciones de comercialización de medicamentos o representantes locales de los mismos, tengan o no actividad productiva. Se corresponden también con el $50 \%$ de los inscritos en el código CNAE 21: Fabricación de productos farmacéuticos en el momento en el que se realizó la encuesta (2015).

Teniendo en cuenta los objetivos de la investigación y los trabajos publicados sobre IC y sobre el sector farmacéutico, se diseñó un cuestionario ad hoc para el objetivo que se perseguía. Se realizó una entrevista presencial con 4 de las empresas integrantes de la muestra para constatar su idoneidad y mejorar las preguntas que pudieran requerir de información sensible en un sector tan hermético como el estudiado.

Después de incorporar las sugerencias y mejoras propuestas por los encuestados en el estudio piloto, se enviaron los cuestionarios finales a todos los miembros de Farmaindustria. Tras cinco meses de seguimiento de la encuesta se recibieron 51 cuestionarios válidos, lo que representa un 27,4 $\%$ de la muestra inicial.

\begin{tabular}{|l|l|}
\hline Muestra & $\begin{array}{l}186 \text { laboratorios miembros de la } \\
\text { asociación Farmaindustria }\end{array}$ \\
\hline Estudio piloto & Febrero 2015, 4 empresas \\
\hline $\begin{array}{l}\text { Envío y seguimiento de la } \\
\text { encuesta }\end{array}$ & Marzo-julio 2015 \\
\hline $\begin{array}{l}\text { Número de cuestionarios } \\
\text { recibidos }\end{array}$ & $51(27,4 \%)$ \\
\hline Casos perdidos & 1 \\
\hline
\end{tabular}

En el siguiente apartado se exponen los principales resultados obtenidos.

\section{Resultados}

Con los datos recibidos se realizó, en primer lugar, un análisis descriptivo sobre las actividades y expectativas de las empresas del sector farmacéutico en el uso de la IC para la cooperación. Por otra parte se realizó un análisis cluster para comprobar si existía más de un patrón de actuación en el uso de la IC con fines colaborativos en el sector. A continuación se exponen los análisis mencionados.

\subsection{Fase 1: Planificación de actividades}

Las empresas necesitan planificar las actividades de IC teniendo en cuenta la finalidad que buscan. La planificación puede necesitar, según los casos, información legal, fiscal, de los competidores o sobre los cambios del mercado, de los productos y procesos, etc.

Para poder medir el interés por la cooperación en esta fase, se preguntó directamente a las empresas de la muestra si recurrían a la IC en los casos en los que una empresa deseaba colaborar con otras. Sólo tres compañías declararon no usar la IC para colaborar con otras entidades.

En esta fase, un 65\% de las empresas que respondieron al cuestionario declararon que utilizan bastante o habitual- mente la IC cuando persiguen realizar actividades de cooperación. Por tanto, en contra de la concepción competitiva de la IC y de acuerdo con la línea que proponen Tena y Comai (2001), los resultados indican la importancia de las actividades de IC para conseguir los objetivos de cooperación de estas empresas.

\subsection{Fase 2: Recogida de información}

Las empresas evalúan dónde y en qué condiciones recogen los datos y la información necesaria para cubrir cada necesidad. Es interesante conocer las fuentes que utilizan para recoger esas informaciones, si son internas, externas, privadas o públicas, estructuradas o no, etc. Una de estas fuentes a analizar son los colaboradores de las compañías. Es fácil recurrir a ellos en busca de información que puede usarse tanto en inteligencia competidora como cooperativa (Ortoll et al., 2010).

En esta fase se preguntó a las empresas farmacéuticas por el uso de "colaboradores" como fuente de información. Se les preguntó diferenciando a empresas colaboradoras filiales de la matriz (fuente interna) y colaboradoras socias en proyectos de investigación ajenos a su organización (fuente externa).

En esta etapa, fase de recogida de información, más del $50 \%$ de las empresas que contestan usan habitualmente las fuentes de información de sus socios en proyectos de investigación, lo que indica el nivel de relación que llegan a tener empresas tan celosas en la seguridad de su información cuando se trata de investigar con otras compañías.

También son altos los porcentajes de las compañías que usan la información de otras filiales del grupo de forma habitual o en bastantes ocasiones (55\%). Considerando que se les preguntaba dentro del contexto de la IC, es destacable que los porcentajes de respuesta para socios y para filiales del mismo grupo no son muy distantes aunque es lógico que se recurra en mayor medida a las filiales como fuente de información que a los socios externos.

\subsection{Fase 3: Análisis y difusión de la información}

En este grupo de actividades hay que destacar, en primer lugar, la importancia de los programas que usan para el análisis de los datos, el control del análisis y el almacenaje tanto de la información como de la inteligencia que se necesita y se genera.

Además, la difusión o diseminación de la inteligencia generada en la organización puede llevarse a cabo teniendo en cuenta a los usuarios individuales o de grupo, de departamentos concretos (marketing, $1+D+i$, red comercial, dirección general, etc.), de filiales o de dentro de la organización. Interesa conocer también el tipo de difusión y por qué medios se realiza (reuniones, intranet u otros).

En esta tercera fase del ciclo de IC, análisis y difusión, se les preguntó a las empresas en qué medida la diseminación de la inteligencia se realizaba entre las filiales de la compañía y en qué medida era enviada a los socios de los proyectos directamente: el $40 \%$ envía la información relevante a los socios de los proyectos y también lo hacen en un porcentaje ligeramente superior (43\%) a las propias filiales. Destaca- 
mos, por tanto, la importancia que se le da a la gestión de la información entre socios cuando se tiene un proyecto en común.

Conviene señalar que no se preguntaba por un proyecto concreto, sino por su actuación habitual en el marco de la IC.

\subsection{Fase 4: Utilización de los resultados}

En este apartado exploramos las actividades encaminadas a resolver los problemas de las empresas. Nos centramos en analizar si las empresas perciben que la IC ha logrado solventar situaciones comprometidas en departamentos, filiales, proyectos, etc., y si se espera que los resultados del proceso de IC sirvan más adelante para otras cuestiones diferentes.

Se preguntó a las empresas farmacéuticas en qué medida los informes generados por la IC sirvieron para solucionar problemas de filiales, matriz u otras empresas en el territorio nacional, en el exterior o con socios de la empresa en proyectos de investigación compartidos.

\section{Diversos organismos recomiendan una mayor cooperación entre las empresas del sector farmacéutico y con las admi- nistraciones públicas}

La otra cuestión sobre cooperación que se formuló, fue si los resultados de la IC serían utilizados en un futuro por las empresas filiales, matriz u otras firmas nacionales o internacionales del grupo, así como por socios de la empresa en proyectos o investigaciones.

En esta fase, la frecuencia de utilización de resultados parece más clara por parte de las firmas del grupo que por socios de proyectos.

Las respuestas ponen de manifiesto que un alto porcentaje (66\%) utiliza con bastante frecuencia los informes generados en IC en otras firmas del mismo grupo, pero no se trata de un proceso sistematizado, sólo en el $10 \%$ de los casos se da esa sistematización. En las dos situaciones descritas disminuye la utilización cuando se les pregunta por las firmas del mismo grupo pero en el extranjero.

La utilización de informes de IC entre socios de proyectos también es importante, no es habitual pero un $40 \%$ de los encuestados consideran que se utiliza bastante. Por el contrario, casi un $39 \%$ no los utiliza nunca o casi nunca.

\subsection{Fase 5: Evaluación del funcionamiento del pro- ceso}

La evaluación de los resultados del ciclo de IC es necesaria para hacer posible la mejora del proceso y los ajustes sobre los objetivos que fueron planteados. Es necesario valorar la flexibilidad de las actividades, la satisfacción de los usuarios de la inteligencia, las mejoras que se hayan o no producido en las áreas estratégicas de la organización, etc.

Aquí, se preguntó a las empresas farmacéuticas si consideraban que el uso de la IC les ayudó en la cooperación con otras entidades, detectando futuros socios en el área de financiación o en futuras colaboraciones para la innovación. Los datos muestran la repercusión del uso de IC para todo ello en el sector. Las compañías farmacéuticas consideran que la IC fomenta la colaboración con otras entidades. En cuanto a la detección de nuevos socios financieros, aproximadamente un $50 \%$ declaró que era bastante o muy importante.

El análisis descriptivo de los datos recogidos pone de manifiesto que tal y como plantean Tena; Comai (2001) y GarcíaAlsina; Ortoll (2012), las empresas consideran la inteligencia competitiva como un método útil para la colaboración con otras empresas y, de hecho, tienen presente esta utilidad en cada una de las fases del proceso.

Se han detectado diferencias de uso de la IC para la cooperación en cada una de las 5 fases anteriores del ciclo y también diferentes grados de interés en la colaboración, probablemente buscando la seguridad de la información y el conocimiento. Sin embargo, existe una actitud muy positiva ante el uso de estas técnicas para encontrar cooperación de otros agentes.

Si tenemos en cuenta que algo más del $70 \%$ de las compañías encuestadas declararon su intención de hacer inversiones en IC antes de un año, entendemos que existe una buena disposición para incrementar los recursos destinados a IC en el sector farmacéutico español.

\subsection{Análisis cluster de los datos}

Con el objetivo de medir el compromiso de las empresas con el ciclo de IC y analizar el nivel de uso de cada una de las 5 etapas, su relación con las actividades de seguridad, el grado de interés en las mejoras internas por parte de los responsables de la IC, y la repercusión de la IC en las actividades de colaboración que lleva a cabo la empresa, se elabora el índice de desarrollo del proceso (IDP).

Este índice permite analizar el nivel de desarrollo del proceso de IC en cada organización. El índice se calcula como el promedio del sumatorio de los valores promedio obtenidos para cada una de las cinco fases. Por su parte, el resultado del promedio de los valores obtenidos para cada una de las variables que integran cada fase, se agrega para generar unos índices parciales $\left(F_{i}\right)$.

$$
I D P=\frac{\sum_{i=1}^{5} F_{i}}{5} \quad \text { donde } \quad F_{i}=\frac{\sum_{j=1}^{n} v_{i j}}{n}
$$

$v_{i j}$ es el valor de las " $n$ " variables que integran la fase " $i$ ".

Una vez calculados los índices generales y parciales se procede a realizar un análisis cluster que nos pueda identificar grupos de comportamiento diverso con respecto al fenómeno de la inteligencia competitiva. Se identifican dos clusters claramente diferenciados.

- El cluster 1 se caracteriza por ser el más grande e incluir el 54\% de los casos (resultados de 27 laboratorios). Todos los laboratorios que lo integran tienen índices parciales altos y el índice total de todos sus componentes es mayor o igual a 3,35. 
- El cluster 2 incluye al resto de los casos y son el 46\% (resultados de 23 laboratorios). Todos los laboratorios que lo integran tienen índices parciales medios-bajos y el índice total (IDP) es para todos los casos inferior a 3,35 (el máximo valor es 3,33 ).

Podríamos decir que el cluster 1 reúne a las empresas que tienen un nivel de compromiso elevado con la inteligencia competitiva mientras que las del cluster 2 parecen haberla aplicado en menor medida.

Una vez obtenidos los dos clusters intentamos determinar si existen diferencias significativas entre ellos en cuanto al uso con fines cooperativos de la inteligencia competitiva.

Para ello se calcula un índice parcial (IPUC = índice promedio de uso de la IC en colaboración) que mide el uso de la inteligencia competitiva con fines de cooperación. El cálculo se realiza de la siguiente forma:

$$
I P U C=\frac{\sum_{t=1}^{6} V C_{t}}{6}
$$

Donde $V C_{i}$ son las variables que muestran la repercusión de la inteligencia competitiva en la colaboración. Se evalúan de 1 a 5 , por lo que el indicador también tomará valores de 1 a 5. Las variables analizadas $\left(V C_{t}\right)$ son:

$V C_{1}$ : Interés en colaboraciones que se apoyan en la IC $V C_{2}$ : Uso de fuentes de colaboradores

$V C_{3}$ : Usuarios externos de resultados compartidos en informes remitidos a filiales y colaboradores

$V C_{4}$ : Uso de resultados en varias organizaciones que conllevan facilitar la resolución de problemas

$V C_{5}$ : Uso externo de resultados por parte de otras organizaciones

$V C_{6}$ : Fomento de la cooperación gracias a las actividades de IC

La media del índice de colaboración así calculado es de 3,87 para el cluster 1 y de 2,31 para el cluster 2 . Para comprobar si esta diferencia es estadísticamente significativa se realiza un T-test para dos muestras independientes. Los resultados (que se muestran en la tabla 1) ponen de manifiesto que existe una diferencia de medias significativa en lo que se refiere al uso de la inteligencia competitiva para la cooperación entre las empresas que tienen mayor y menor compromiso con la misma.
Así, comprueban dos formas de actuación de las empresas del sector apoyadas en un análisis cluster. Se identifica un primer grupo de empresas que puntúan alto en todos los subíndices y en el índice general elaborado, mientras que el otro grupo presenta puntuaciones medias o bajas en todos ellos. Es decir, cuando se trata de comprobar si existe un comportamiento característico de cada uno de los clusters en lo que se refiere al uso de la inteligencia competitiva para la cooperación, se comprueba que existen diferencias significativas en la media entre los dos grupos en lo que se refiere al índice general de cooperación (IDP).

\section{Entre otras razones, los laboratorios buscan la cooperación para luchar con- tra los medicamentos genéricos}

\section{Conclusiones}

Después de analizar los resultados de la encuesta podemos concluir, como idea principal, que la mayoría de las empresas farmacéuticas apuestan por compartir información, conocimiento e IC con otras empresas del sector, filiales o socias en proyectos de cooperación e investigación, a pesar de su habitual hermetismo. Las empresas farmacéuticas buscan la cooperación, como puede observarse en los acuerdos realizados en el sector en los últimos meses, con el fin de compensar la aparición de los fármacos genéricos, para investigar en oncología, enfermedades raras, Alzheimer, vacunas o, incluso, intercambiar activos.

Parece que si la apuesta es por la cooperación, las empresas están encontrando en el proceso de inteligencia un aliado muy valioso para avanzar hacia sus objetivos de investigación y crecimiento. Es importante que las empresas del sector farmacéutico reconsideren las actividades de IC de forma que se mejore su eficiencia en la colaboración, sea cual sea el área en la que se realice.

Por otra parte, se hace necesario que los profesionales de la inteligencia revisen su gestión para potenciar redes de cooperación que faciliten la consecución de los objetivos corporativos de forma eficiente. Las decisiones tales como el nivel de compromiso con los socios o de confidencialidad de la información, los sistemas de protección de la información y las garantías de seguridad que se usarán, etc., determinarán a la vez el avance tanto de la IC como de las relaciones de cooperación entre las empresas del sector.

Tabla 1. Diferencia de medias para el índice general de cooperación (IDP): prueba de muestras independientes

\begin{tabular}{|c|c|c|c|c|c|c|c|c|c|c|}
\hline & & \multicolumn{2}{|c|}{$\begin{array}{l}\text { Test Levene de igual- } \\
\text { dad de varianzas }\end{array}$} & \multicolumn{7}{|c|}{ Prueba T para la igualdad de medias } \\
\hline & & \multirow{2}{*}{$\mathrm{F}$} & \multirow{2}{*}{ Sig. } & \multirow[b]{2}{*}{$\mathrm{t}$} & \multirow{2}{*}{ gl } & \multirow{2}{*}{$\begin{array}{l}\text { Sig. (bila- } \\
\text { teral) }\end{array}$} & \multirow{2}{*}{$\begin{array}{l}\text { Dif. de } \\
\text { medias }\end{array}$} & \multirow{2}{*}{$\begin{array}{l}\text { Error } \\
\text { típico de } \\
\text { la dif. }\end{array}$} & \multicolumn{2}{|c|}{ IC del 95\% de la dif. } \\
\hline & & & & & & & & & Inferior & Superior \\
\hline \multirow{2}{*}{$\begin{array}{l}\text { Colaboración } \\
\text { total }\end{array}$} & $\begin{array}{l}\text { Varianzas } \\
\text { iguales }\end{array}$ & 0,354 & 0,554 & 9,348 & 48,000 & 0,000 & 1,56688 & 0,16762 & 1,22986 & 1,90389 \\
\hline & $\begin{array}{l}\text { Varianzas no } \\
\text { iguales }\end{array}$ & & & 9,277 & 45,045 & 0,000 & 1,56688 & 0,16890 & 1,22671 & 1,90704 \\
\hline
\end{tabular}




\section{Bibliografía}

Antia, Kersi-Darius; Hesford, James (2007). "A processoriented view of competitive intelligence and its impact on organizational performance". Journal of competitive intelligence and management, v. 4, n. 1, pp. 5-33.

Arroyo-Varela, Silvia-Regina (2005). Inteligencia competitiva. Una herramienta clave en la estrategia empresarial. Madrid: Ed. Pirámide. ISBN: 9878436819268

Ashton, William-Bradford; Stacey, Gary S. (1995). "Technical intelligence in business: understanding technology threats and opportunities". Journal of technology management, v. 10, n. 1.

https://doi.org/10.1504/IJTM.1995.025615

Baiget, Tomàs (1999). "Uso de la información en laboratorios farmacéuticos". El profesional de la información, v. 8, n. 12, pp. 15-21.

http://www.elprofesionaldelainformacion.com/contenidos/1999/ diciembre/uso_de_informacion_en_laboratorios farmaceuticos. html

Bernhardt, Douglas (1994). "I want it fast, factual, actionable: Tailoring competitive intelligence to executives' needs". Long Range Planning, v. 27, n. 1, pp 12-24.

http://www.oss.net/dynamaster/file_archive/040320/ effb2b42b78bf84b091c680e7949110c/OSS1994-02-03.pdf

Cavaller, Víctor (2009). "Actualidad de la inteligencia competitiva". Cuadernos de inteligencia competitiva, vigilancia estratégica, científica y tecnológica, v. 2, Edición 1, pp. 31-44. http://www.raco.cat/index.php/QUICVECT_es/article/ view/123706/171653

Cetisme (2003). Inteligencia económica y tecnológica. Guía para principiantes y profesionales. Servicio de publicaciones de la Comunidad de Madrid.

http://www.fundacionede.org/gestioninfo/docs/contenidos/_ inteligenciaeconomicamadrid_.pdf

Choo, Chun-Wei (2002). Information management for the intelligent organization: The art of scanning the environment. Medford, NJ: Information Today. ISBN: 1573871257

Cohen, Sacha (1997). "Digital detective". Training \& development, v. 51, n. 6, pp. 16-19.

Escorsa, Pere; Maspons, Ramón (2001). De la vigilancia tecnológica a la inteligencia competitiva. Madrid: Prentice Hall. ISBN: 9788420530573

García-Alsina, Montserrat; Ortoll-Espinet, Eva (2012). La inteligencia competitiva. Evolución histórica y fundamentos teóricos. Gijón: Ed. Trea SL. ISBN: 9788497046916

Herring, Jan-P. (1999). "Key intelligence topics: A process to identify and define intelligence needs" Competitive intelligence review, v. 10, n. 2, pp. 4-10.

https://legacy.wlu.ca/documents/22437/04_Herring_ KITs_A_Process_to_Identify.pdf

Hidalgo-Nuchera, Antonio; León-Serrano, Gonzalo; PavónMorote, Julián (2002). La gestión de la innovación y la tecnología en las organizaciones. Madrid: Eds. Pirámide. ISBN: 9788436817027
Hidalgo-Nuchera, Antonio; Iglesias-Pradas, Santiago; Hernández-García, Ángel (2009): “Utilización de las bases de datos de patentes como instrumento de vigilancia tecnológica". El profesional de la información, v. 18, n. 5, pp. 511518.

https://doi.org/10.3145/epi.2009.sep.04

Hussey, David; Jenster, Per (1999). Competitor intelligence: Turning analysis into success. Wiley series in practical strategy, Ed. Wiley, 222 pp. ISBN: 9780471984078

Jakobiak, François (1992). Exemples commentés de veille technologique. Paris: Les Éditions d'Organisation. ISBN: 978 2708114760

Lesca, Nicolas; Caron-Fasan, Marie-Laurence (2006). Veille anticipative: une autre approche de l'intelligence économique. Paris: Hermès Science Publications. ISBN: 978 2746214590

Martinet, Bruno; Marti, Yves-Michel (1995). L'intelligence économique: les yeux et les oreilles de l'entreprise. Paris: Les Éditions d'Organisation. ISBN: 9782708118171

McGonagle, John J.; Vella, Carolyn M. (1998). Protecting your company against competitive intelligence. Westport, Connecticut: Quorum Books. ISBN: 1567201172

Nalebuff, Barry J.; Brandenburger, Adam M. (1997). “Coopetition: Competitive and cooperative business strategies for the digital economy". Strategy \& leadership, v. 25, n. 6 , pp. 28-35.

https://doi.org/10.1108/eb054655

OMC (2014). Informe sobre el sector farmacéutico, 2014. Organización Médica Colegial de España. Consejo General de Colegios Oficiales de Médicos de España.

https://www.cgcom.es/sites/default/files/informe_omc_ sector_farmaceutico_oct_2014.pdf

Ortoll-Espinet, Eva; López-Borrull, Alexandre; Canals, Agustí; García-Alsina, Montserrat; Cobarsí-Morales, Josep (2010). "El papel del capital social en la inteligencia competitiva". Revista general de información y documentación, v. 20, pp. 313-323.

https://revistas.ucm.es/index.php/RGID/article/download/ RGID1010110313A/9062

Palop, Fernando; Vicente, José M. (1999). Vigilancia tecnológica e inteligencia competitiva. Su potencial para la empresa española. Fundación Cotec.

http://docplayer.es/6739015-Vigilancia-tecnologica-einteligencia-competitiva-su-potencial-para-la-empresaespanola.html

Prescott, John E. (1999). "The evolution of competitive intelligence: designing a process for action". Proposal management, Spring, pp. 37-52.

http://bit.ly/2bH8d5Y

Prescott, John E. (2003). "The social capital of Cl professional". Competitive intelligence magazine, v. 6, n. 1, pp. 33-35. http://www.scip.org/?page=scippublications

Salvetat, David; Le-Roy, Frédéric (2007). "Coopétition et intelligence économique". Revue française de gestion, v. 33, n. 176, pp. 147-161. 
https://www.cairn.info/revue-francaise-de-gestion-2007-7page-147.htm

SCIP (1999). "List of the Cl cycle as published". Society of Competitive Intelligence Professionals.

http://www.scip.org

Tena, Joaquín; Comai, Alessandro (2001). “Los propósitos de la inteligencia en la empresa: competidora, cooperativa, neutral e individual". El profesional de la información, v. 10, n. 5, pp. 4-10.

http://www.elprofesionaldelainformacion.com/contenidos/2001/ mayo/1.pdf

Tena, Joaquín; Comai, Alessandro (2003). "La inteligencia competitiva en la planificación estratégica y financiera". Harvard Deusto finanzas y contabilidad, n. 56, pp. 30-37.
https://www.academia.edu/20788546/La_IC_en_la planificacion_estrategica_y_financiera_-_Harvard-Deusto

Tena, Joaquín; Comai, Alessandro (2005). "El desarrollo de la inteligencia competitiva en España: Un recorrido bibliográfico". Puzzle, año 4, n. 16. marzo-abril, pp. 4-10. http://www.miniera.es/revista?page $=1$

Trim, Peter R. J. (2004). "The strategic corporate intelligence and transformational marketing model". Marketing inteIligence \& planning, v. 22, n. 2, pp. 240-256.

https://doi.org/10.1108/02634500410525896

Vignettes, Mario (2010). "El ciclo de inteligencia: naturaleza y alternativas". Inteligencia y seguridad, n. 8, pp. 113-136. http://libros-revistas-derecho.vlex.es/vid/ciclo-inteligencianaturaleza-alternativas-459760210

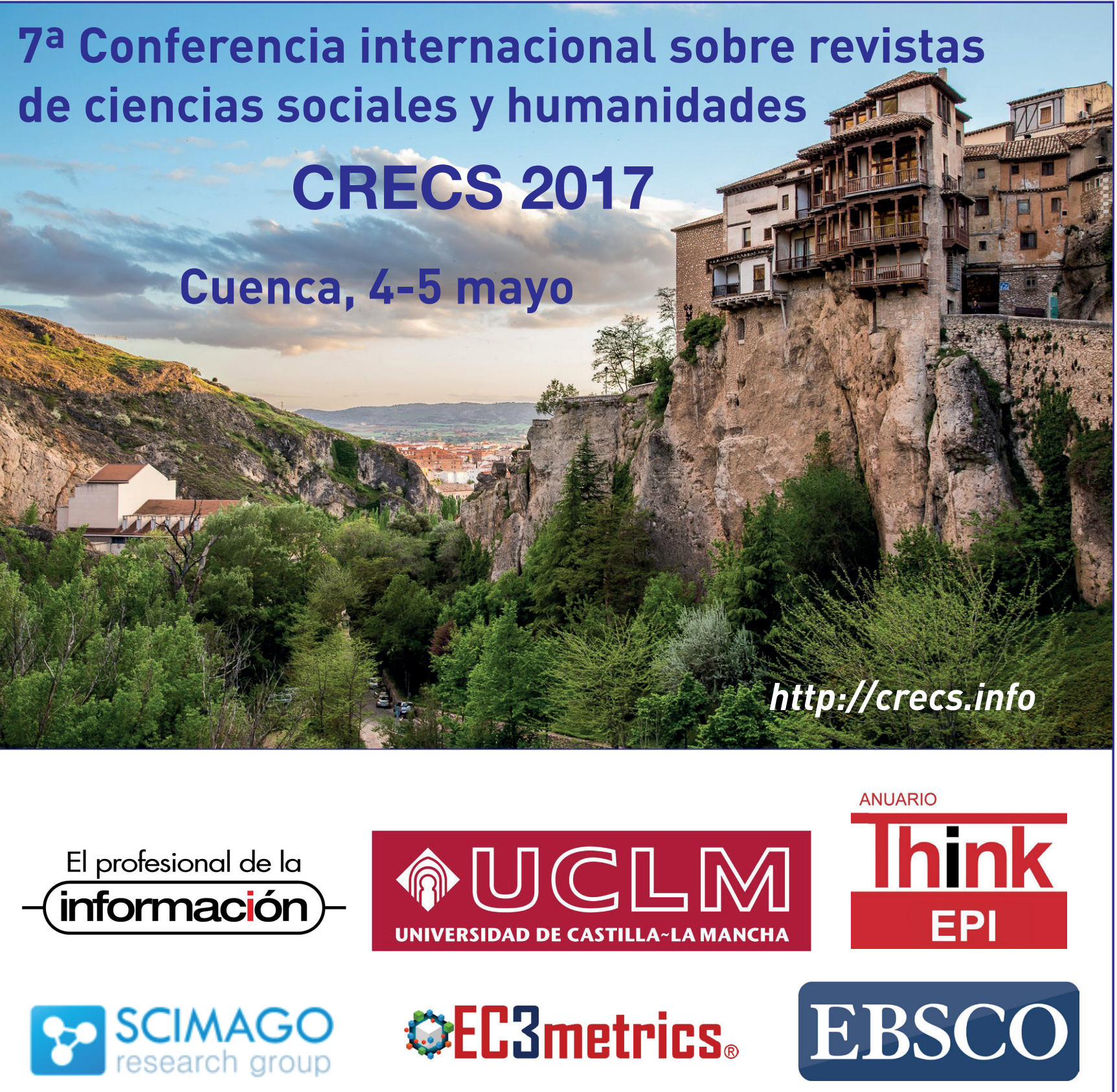

